\title{
A new solution technique for cathodic protection systems with homogeneous region by the boundary element method
}

\author{
W. J. Santos ${ }^{a}$ (D) , S. L. D. C. Brasil ${ }^{b}$, J. A. F. Santiago (1) ${ }^{c}$ and J. C. F. Telles ${ }^{c}$ \\ ${ }^{a}$ Department of Mathematics, UFRRJ, Seropedica, Brazil; ${ }^{b}$ School of Chemistry, UFRJ, Rio de Janeiro, \\ Brazil; ' ${ }^{C}$ Department of Civil Engineering, COPPE/UFRJ, Rio de Janeiro, Brazil
}

\begin{abstract}
The purpose of this work is to efficiently evaluate the design of cathodic protection (CP) systems of tank bottoms using concentric ring or linear anodes. As customary in current $\mathrm{CP}$ systems, the outer surface of the tank bottom is usually in electrical contact with a slender homogeneous layer of conductive concrete (or something similar) which in turn is in direct contact with the homogeneous deep soil region. The boundary element method (BEM) together with a subregion technique has been widely adopted to analyse such CP systems where the domain consists of two (or even more) homogeneous zones. However, the numerical solution of the final matrix system of equations can be quite time-consuming, especially if the slender intermediate layer is to be discretised, requiring a considerable number of elements, due to its somewhat reduced thickness. To overcome this problem, the present work proposes a new methodology in which the slender subregion is indirectly introduced, as a theoretically created polarisation curve, acting as a new boundary condition at the boundary of the soil domain (original common interface). Numerical simulations have been carried out using BEM implementations and results are discussed, including CP studies of practical axisymmetric and three-dimensional engineering problems.
\end{abstract}

\section{ARTICLE HISTORY}

Received 21 September 2017

Accepted 3 November 2017

\section{KEYWORDS}

Axisymmetric problem; nonlinear boundary conditions; subregions; three-dimensional problem

\section{Introduction}

Cathodic protection (CP) is an electrochemical technique used to prevent or to reduce corrosion rate of metallic structures. Electrochemical systems require anode, cathode, electric circuit and electrolyte to promote the current flux between anode and cathode. Thus, cathodic protection can be applied to buried, submerged or, less frequently, concrete structures. The protection of the structure against corrosion can be achieved by making it the cathode of an electrochemical cell. Thermodynamically, steel is immune to corrosion processes

CONTACT J.C.F.Telles telles@coc.ufrj.br 
when the electrochemical potential achieves a predetermined value, as prescribed by specific standards (ISO/15589-1, 2015; NACE/SP0169, 2007).

In practice, the goal is to provide a uniform potential distribution on the metal surfaces, limited by a minimum potential value to guarantee protection from the corrosion using an external power source (impressed current CP) or a galvanic anode (Fontana \& Greene, 1967; Roberge, 1999). The concept of this technique is widely discussed in the literature (Peabody, 2001). Galvanic or impressed current are used to protect structures such as offshore platforms, ships, buried pipelines and storage tanks.

Considering aboveground storage tanks, internal and external corrosion processes can occur. To prevent internal corrosion, galvanic anodes can be placed on the bottom of the tank and to protect the underside (soil side), impressed current is an option. Effective cathodic protection will increase reliability and reduce costs associated with maintenance and inspection. Installation of secondary spill containment, motivated by past tank failures and associated environmental damage, is currently required for new tank installations (USEPA, 1988). Cathodic protection of tanks with secondary spill containment can be achieved using anodes placed between the liner and the tank bottom (Koszewski, 1999). In addition, a common configuration includes the use of a slender homogeneous medium, as sand/bentonite mixtures or conductive concrete, for example, between the tank and the homogeneous deep soil region.

The traditional cathodic protection systems design consists of the application of empirical formulas to determine the current density required. The great advantage in applying numerical techniques to analyse and optimise cathodic protection systems is the possibility of considering the actual geometry of the structures. The current density and potential distributions are influenced not only by the electrolyte resistivity, but also by the structure geometry.

The most common methods for modelling cathodic protection systems are the finite element method (FEM) and the boundary element method (BEM). Mathematical simulations of cathodic protections systems using FEM can be seen in Montoya, Aperador, and Bastidas (2009), Montoya, Gakvan, and Genesca (2011), Parsa, Allahkaram, and Ghobadi (2010). Due to its accuracy and simplicity of mesh generation, the BEM is the most appropriate technique to solve problems involving CP systems (Riemer \& Orazem, 2010). This method requires only the representation of anodes and cathodes surfaces, which may lead to better resolution and reduction in computer run time. Several different applications of BEM to study CP systems have been reported in the literature, including reference to practical analyses performed by offshore oil companies (BEASY, 2000; Brasil, Telles, \& Miranda, 1991; Kim, Kim, Choi, Lim, \& Kim, 2017; Santiago \& Telles, 1997; Telles, Mansur, Wrobel, \& Marinho, 1990). Recently, the method of fundamental solution (MFS) has also been used successfully to CP problems (Santos, Santiago, \& Telles, 2012, 2014, 2016). 
Polarisation curves define the electrochemical conditions of a metallic surface in an electrolyte, considering the relation between current density and potential. The good correlation between in-situ measurements and numerical results largely depends on the appropriate prescription of boundary conditions that are based on experimental tests carried out in laboratory. Therefore, polarisation curves and resistivity values, applied as problem conditions, need to be carefully determined to guarantee accurate results. The BEM implementation includes a Newton-Raphson solution algorithm to accommodate the nonlinear boundary conditions given by such polarisation curves (Azevedo \& Wrobel, 1988).

The numerical solution of the matrix system obtained with the BEM is a time consuming step of the technique, and hence can be a burden in presence of large number of elements. In Kita and Kamiya (1994), this disadvantage was overcome transforming the linear system for each subregion into equations similar to the stiffness equations of the FEM, and then the global matrix equation can be constructed by superposition of these equations for each subregion. Nevertheless, a relatively large global coefficient matrix was still needed. $\mathrm{Lu}$ and $\mathrm{Wu}$ (2005) proposed a new subregion boundary element technique based on the domain decomposition method. The technique is more efficient than traditional methods because it significantly reduces the size of the final matrix, being applicable to the stress analysis of multi-region elastic media, such as layered materials.

Storage tanks can be located on top of a slender homogeneous layer of conductive concrete which in turn is in direct contact with the homogeneous deep soil region. In this scenario, the BEM procedure together with a subregion technique can be used to analyse the multi-region CP system. The present work proposes a new methodology in which a theoretically created polarisation curve, to be introduced at the common interface of adjacent zones, is obtained. This allows for a standard single region BEM solution procedure to be applied, without need to discretise the slender subregion. After obtaining the potential and current density at the original concrete/soil interface, the potential distribution can be easily obtained over the actual tank bottom by an inversion of the theoretical process used for getting the modified polarisation curve. Results of applications are discussed to demonstrate the accuracy and efficiency of the method, involving bottoms of storage tanks.

\section{Mathematical model}

In order to design $\mathrm{CP}$ systems for tank bottoms, one needs to know the electrochemical potential and current density over the metal to be protected. As can be seen in Figure 1, the tank bottom is usually in electrical contact with a slender conductive concrete layer, of low resistivity $\rho_{1}$, which in turn is in direct contact with a homogeneous deep soil region of resistivity $\rho_{2}$. Furthermore, CP is achieved using anodes placed between the liner and the slender conductive layer. 


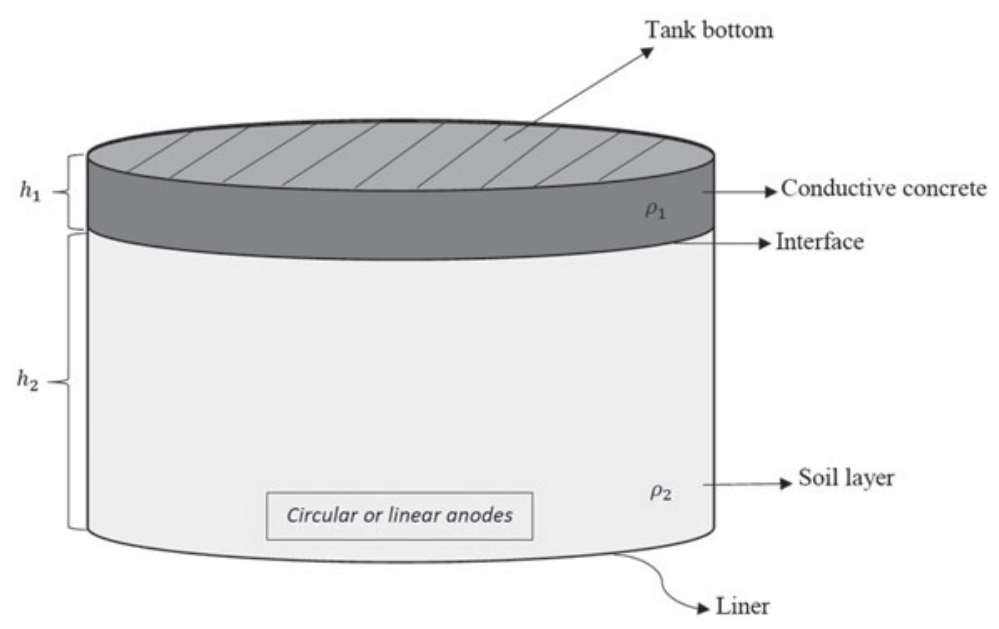

Figure 1. Hypothesis of the potential problem.

The conductive concrete layer height is represented as $h_{1}$, whereas the height of the soil region is denoted by $h_{2}$.

Considering that the CP technique includes the two subregions of Figure 1, the mathematical model of the problem is based on a Laplace equation for the electrochemical potential $(\phi)$ :

$$
\nabla^{2} \phi=0
$$

Over the metal surface in direct contact with the conductive concrete, the boundary condition is given by the polarisation curve, which describes a nonlinear relationship between current density $(i)$ and electrochemical potential on the metal, $\phi=f(i)$. All the other boundaries are insulated $\left(i=0 \mathrm{~A} / \mathrm{m}^{2}\right)$. In addition, from Ohm's law

$$
i=k \frac{\partial \phi}{\partial n}
$$

in which $k$ is the conductivity of the electrolyte and $\mathbf{n}$ is the outward normal to the boundary $\Gamma$.

The starting equation to represent the electrical field problem using boundary elements (Brebbia, Telles, \& Wrobel, 1984) is

$$
c(\xi) \phi(\xi)=\int_{\Gamma} \phi^{*}(\xi, x) i(x) \mathrm{d} \Gamma-\int_{\Gamma} i^{*}(\xi, x) \phi(x) \mathrm{d} \Gamma,
$$

where $\phi^{*}(\xi, x)$ and $i^{*}(\xi, x)$ define the fundamental solution for potential and respective current density. The coefficient $c(\xi)$ depends on the boundary geometry at the source point $\xi$.

In order to solve numerically Equation (3), the boundary $\Gamma$ is to be discretised into a number of elements whose geometry, current and potential are approximated as functions of the nodal values. Thus, the familiar algebraic system can be obtained (Brebbia et al., 1984): 


$$
\mathbf{H} \phi=\mathrm{Gi} .
$$

For nonlinear boundary conditions, system (4) is usually solved by a tangent Newton-Raphson method, which can be formulated considering only the firstorder terms in a Taylor series expansion:

$$
\mathbf{i}^{k}=\mathbf{i}^{k-1}+\mathbf{J}^{k-1} \boldsymbol{\phi}^{k},
$$

where the Jacobian matrix $\mathbf{J}$ is given by

$$
\mathbf{J}^{k-1}=\left(\frac{\partial \mathbf{i}}{\partial \boldsymbol{\phi}}\right)^{k-1} .
$$

The axisymmetric fundamental solution for Laplace's equation is used to simulate the $\mathrm{CP}$ system of tank bottoms using concentric rings. In this case, the fundamental potential can be calculated explicitly in terms of the complete elliptic integral of the first kind $K(m)$ as (Brebbia et al., 1984)

$$
\phi_{\text {axis }}^{*}=\int_{0}^{2 \pi} \phi^{*}(\xi, x) \mathrm{d} \theta=\frac{4 K(m)}{(a+b)^{\frac{1}{2}}},
$$

where $\phi^{*}$ is the three-dimensional fundamental solution in cylindrical polar coordinates $(R, \theta, Z)$ and

$$
\begin{aligned}
m & =\frac{2 b}{a+b}, \\
a & =R^{2}(\xi)+R^{2}(x)+[Z(\xi)-Z(x)]^{2}, \\
b & =2 R(\xi) R(x) .
\end{aligned}
$$

The parameter $m$ has range of $[0,1]$. The normal derivative of the axisymmetric fundamental solution is given by

$$
\begin{aligned}
i_{a x i s}^{*}= & \frac{4}{(a+b)^{\frac{1}{2}}} \\
& \cdot\left\{\frac{1}{2 R(x)}\left[\frac{R^{2}(\xi)-R^{2}(x)+[Z(\xi)-Z(x)]^{2}}{a-b} E(m)-K(m)\right] n_{R}(x)\right. \\
& \left.+\frac{Z(\xi)-Z(x)}{a-b} E(m) n_{z}(x)\right\},
\end{aligned}
$$

where $E(m)$ is the complete elliptic integral of the second kind.

For convenience of numerical implementation, the complete elliptic integrals are approximated by polynomial expressions (Abromowitz \& Stegun, 1965). Substituting Equations (7) and (9) into Equation (3), written in cylindrical polar coordinates, and using a collocation technique, it is possible to obtain the system of Equations (4). 

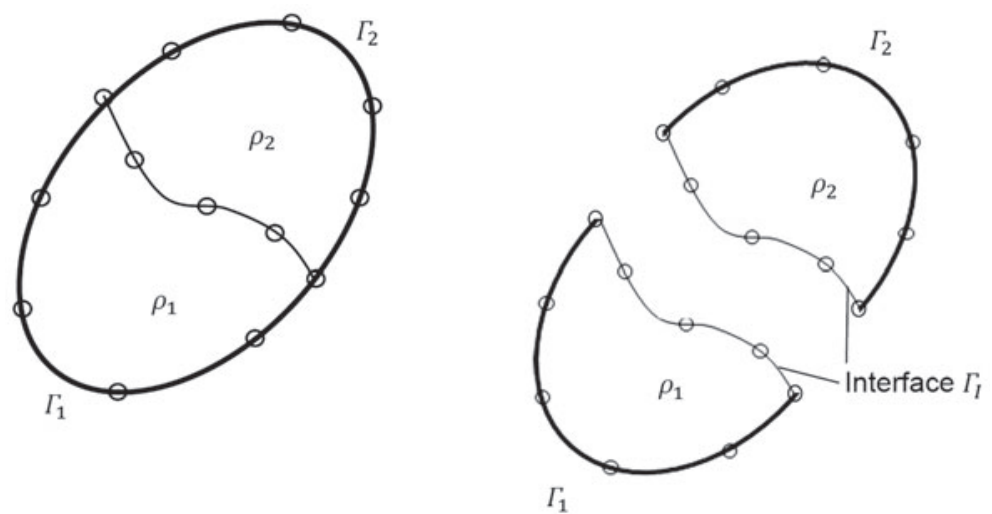

Figure 2. Domain divided into two subregions.

\section{Traditional solution technique}

The potential problem cited above and illustrated in Figure 1 is defined over a piecewise homogeneous body consisting of two subregions and, therefore, the boundary element procedure can be applied to each homogeneous subregion (see Figure 2). The final set of equations for the whole region can then be obtained by assembling the set of Equations (4) for each subregion using compatibility of potentials and equilibrium of fluxes between the common interfaces (Brebbia \& Dominguez, 1989; Brebbia \& Walker, 1980).

The problem under consideration is usually solved using the BEM procedure with a subregion technique. In this case, a large number of elements is required in order to discretise the slender subregion in direct contact with the tank bottom. For this reason, the purpose of the present paper is to obtain a theoretically created polarisation curve, acting as a new boundary condition at what was the original common interface.

\section{A new solution technique}

In order to create the theoretical polarisation curve on the interface, the current flowing through the slender conductive concrete was considered as a through layer thickness linear circuit (resistivity $\rho_{1}$ ). According to Ohm's law, there is a linear relationship between the voltage drop across a circuit element and the current flowing through it. In equation form, this relation can be written as follows:

$$
V=I R,
$$

where $V$ is the potential difference $\left(\phi_{1}-\phi_{2}\right)$ between any two points, in volts $(\mathrm{V}), I$ is the current flowing through, in Amperes (A), and $R$ is the resistance, in ohms $(\Omega)$, as shown in Figure 3. Considering a homogeneous and isotropic material with resistivity $\rho_{1}$, a cross-sectional area $A$ and the height $h_{1}$, the current 


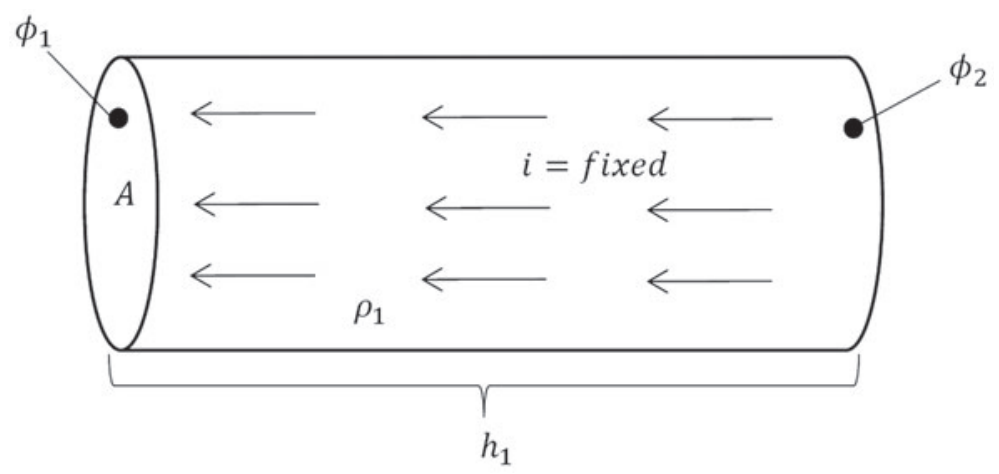

Figure 3. Potential difference between any two points in a circuit.

density and the resistance are given, respectively, by the following equations:

$$
\begin{aligned}
i & =\frac{I}{A}, \\
R & =\rho_{1} \frac{h_{1}}{A} .
\end{aligned}
$$

Using the Equations (11) and (12), it is possible to rewrite the relation (10) for any two points presented in Figure 3 as

$$
\phi_{1}-\phi_{2}=i \rho_{1} h_{1}
$$

After experimentally determining the polarisation curve for the tank bottom, which is in electric contact with the conductive concrete, Equation (13) can be used to create a theoretical polarisation curve for the concrete/soil interface. For each pair of points $\left(i^{k}, \phi_{1}^{k}\right)$ given by experimental polarisation curve over the tank bottom, the corresponding theoretical polarisation curve in the concrete/soil interface is approximated by the point pairs $\left(i^{k}, \phi_{2}^{k}\right) \equiv\left(i^{k}, \phi_{1}^{k}-i^{k} \rho_{1} h_{1}\right)$. Thus, only the soil layer of resistivity $\rho_{2}$ needs to be considered and hence a standard single region BEM procedure can be applied. Finally, after calculating the potential at the boundary that was the original interface, the inversion relation $\phi_{1}^{k}=\phi_{2}^{k}+i^{k} \rho_{1} h_{1}$ is used to determine the potential distribution on the tank bottom.

\section{Numerical results}

\subsection{Boundary condition}

A polarisation curve is a plot of current density $(i)$ versus electrode potential $(\phi)$ for a specific electrode-electrolyte combination. Plots of $\log |i| v s . \phi$ or $v s .(\phi-$ $\phi_{o}$ ) are the basic kinetic law for any electrochemical reaction.

In order to use polarisation curves as boundary conditions, experimental tests should be carried out as close as possible to the real conditions of the cathodic 


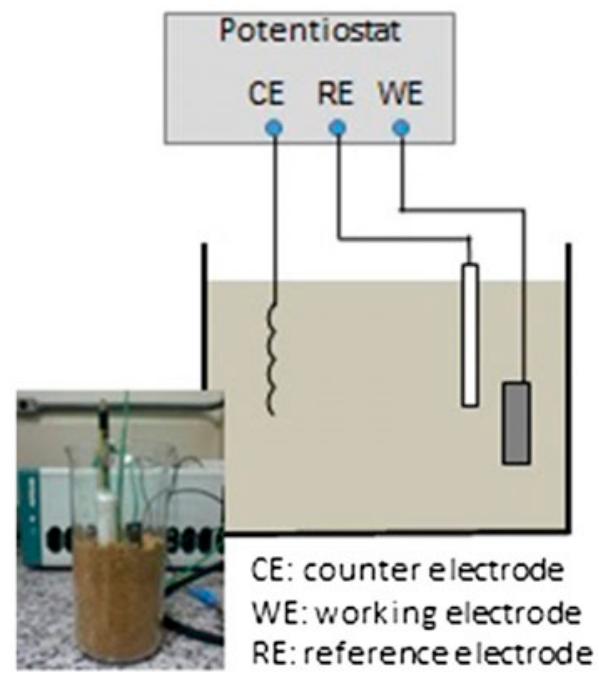

Figure 4. Experimental device to obtain polarisation curve, considered as boundary condition.

protection system under study. Figure 4 shows the experimental device used to obtain the relation between these parameters (current density and potential). A common methodology includes a three-electrode cell: a steel coupon as a working electrode, a reference electrode and a counter-electrode (or an auxiliary electrode).

\subsection{Two-dimensional problem}

For the purpose of testing the proposed methodology, a tilted anode cathodic protection system, shown in Figure 5, was initially analysed. The sacrificial anodes are mathematically represented by linear sources producing a current intensity of $-0.06 \mathrm{~A} / \mathrm{m}$. The idea was to achieve a potential distribution on the metal surface below the minimum potential: $\phi \leq \phi_{c}=-0.850 \mathrm{~V}$ ( $v s$. SCE). The two zones present resistivities $\rho_{1}=80 \Omega \mathrm{m}$ and $\rho_{2}=500 \Omega \mathrm{m}$. The height of the first subregion is $0.5 \mathrm{~m}$.

Table 1 presents the experimental polarisation curve on the metal $\left(i \times \phi_{1}\right)$ and the corresponding theoretical polarisation curve $\left(i \times \phi_{2}\right)$, estimated for the interface location and obtained by relation $\left(i^{k}, \phi_{2}^{k}\right) \equiv\left(i^{k}, \phi_{1}^{k}-i^{k} \rho_{1} h_{1}\right)$.

The proposed formulation is compared with the alternative subregion technique. Figure 6 shows the potential distribution obtained on the metal surface using the subregion technique and the proposed methodology, where the similarity of the results can be seen.

Using the proposed methodology, the process time was approximately one fourth of the subregions approach. In applications involving optimisation problems and inverse problems, this difference will be a lot more significant, due to the necessity of recursively solving the numerical problem. In addition, for this 


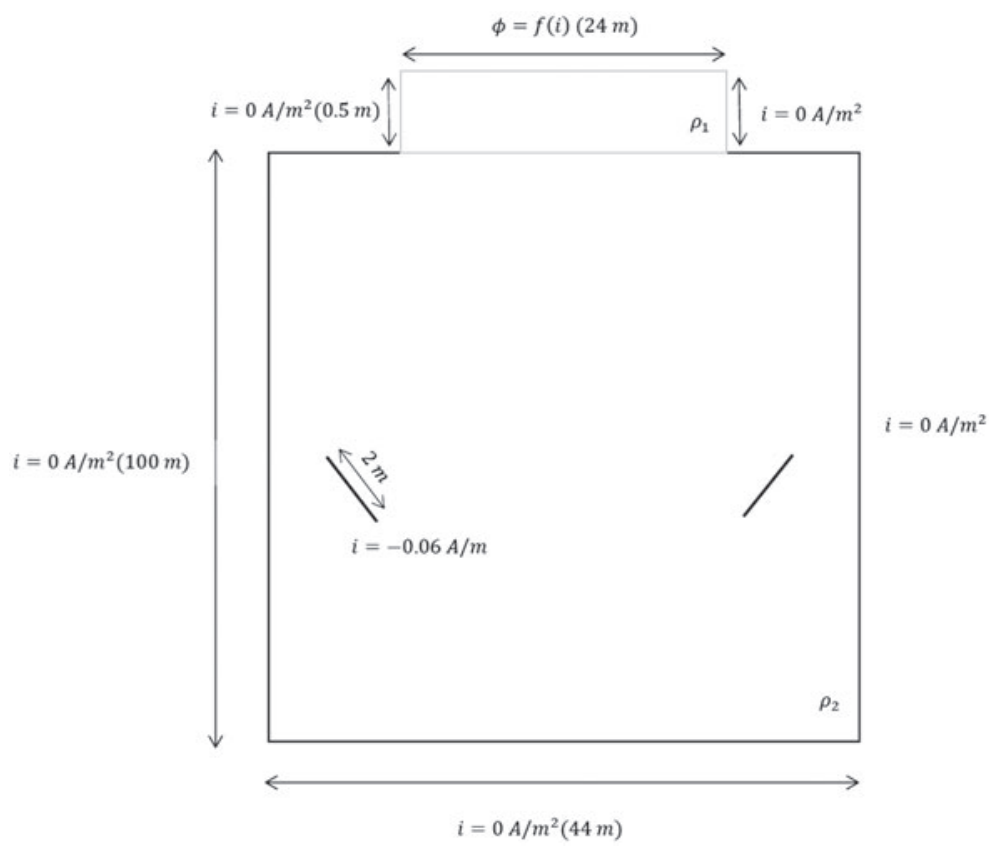

Figure 5. A two-dimensional potential problem.

Table 1. Polarisation curves for the two-dimensional problem.

\begin{tabular}{lcc}
\hline Current density $\left(\mathrm{A} / \mathrm{m}^{2}\right)$ & Potential on the metal $(\mathrm{V})$ & Potential in the interface $(\mathrm{V})$ \\
\hline 0.0022 & -0.835 & -0.923 \\
0.0062 & -0.850 & -1.098 \\
0.0141 & -0.874 & -1.438 \\
0.0192 & -0.881 & -1.649 \\
0.0273 & -0.903 & -1.995 \\
0.0452 & -0.945 & -2.753 \\
0.0570 & -0.984 & -3.264 \\
0.0780 & -1.033 & -4.153 \\
0.1554 & -1.086 & -7.302 \\
\hline
\end{tabular}

example, the geometry was even simplified, avoiding the re-entrant corners of the original problem.

\subsection{Axisymmetric problem}

In this application, the cathodic protection of an external tank bottom (see Figure 1 ) is analysed. Here, a conductive concrete layer with height $h_{1}=0.03 \mathrm{~m}$ and resistivity $\rho_{1}=87 \Omega \mathrm{m}$ is considered and the resistivity of the soil layer with height $h_{2}=0.3 \mathrm{~m}$ is $\rho_{2}=331.34 \Omega \mathrm{m}$. Aiming at reducing corrosion and extending the service life of the tank bottom, a system of concentric ring anodes is proposed. The number of such rings usually varies with the tank diameter. In this case, the diameter of the tank bottom is $84 \mathrm{~m}$ and the fixed distance between the 14 anodes is $3 \mathrm{~m}$. In addition, these anodes are localised over the 


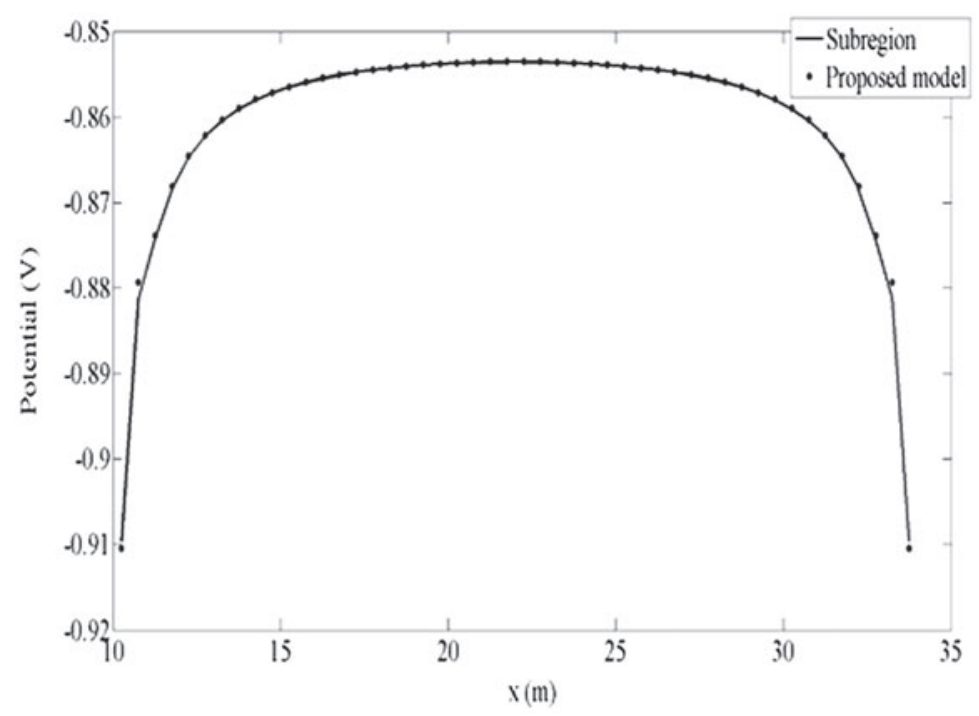

Figure 6. Potential distribution on the metal.

Table 2. Polarisation curves for the axisymmetric problem.

\begin{tabular}{lcc}
\hline Current density $\left(\mathrm{A} / \mathrm{m}^{2}\right)$ & Potential on the metal $(\mathrm{V})$ & Potential in the interface $(\mathrm{V})$ \\
\hline 0.000001 & -0.738 & -0.738 \\
0.000500 & -0.768 & -0.769 \\
0.000600 & -0.818 & -0.820 \\
0.000800 & -0.918 & -0.920 \\
0.001000 & -0.938 & -0.941 \\
0.002000 & -1.018 & -1.023 \\
0.003000 & -1.068 & -1.076 \\
0.006000 & -1.168 & -1.184 \\
0.020000 & -1.318 & -1.370 \\
\hline
\end{tabular}

liner and each anode has a prescribed potential of $-1.6 \mathrm{~V}$. Figure 7 illustrates this somewhat new design procedure to protect tank bottoms.

Table 2 presents the polarisation curve for the tank bottom, which is in electric contact with the concrete, including the theoretical polarisation curve, to be used at the original interface, estimated by relation (13).

The problem has been solved considering just the soil layer and the estimated polarisation curve at the interface. All boundary values have axial symmetry and consequently all domain values are also axisymmetric. Thus, an axisymmetric analysis $(R, Z)$ has been considered in this example, using the axisymmetric fundamental solutions, Equations (7) and (9). The calculated potential values on the metal, solved by proposed formulation and the alternative subregion technique are presented in Figure 8, whereas Figure 9 depicts the BEM solution in the soil layer (electrolyte of resistivity $\rho_{2}$ ). 


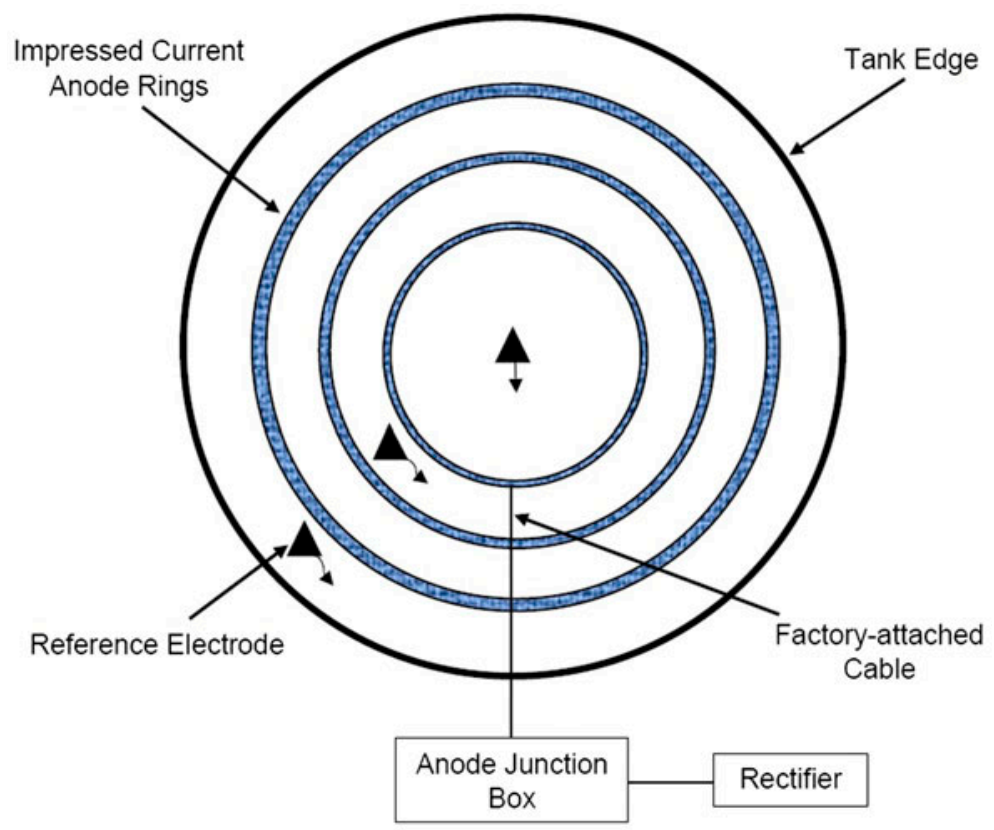

Figure 7. Concentric ring anode system.



Figure 8. Potential distribution on the metal.

The computer run time significantly reduces using the proposed technique in this example. The CPU times were $78.0 \mathrm{~s}$ using BEM with the subregion idea and $5.6 \mathrm{~s}$ using BEM with the tested methodology.

\subsection{Three-dimensional problem}

The last example has been selected to illustrate an actual real size tank bottom application, in which in-situ measurements have been effected. A total of 


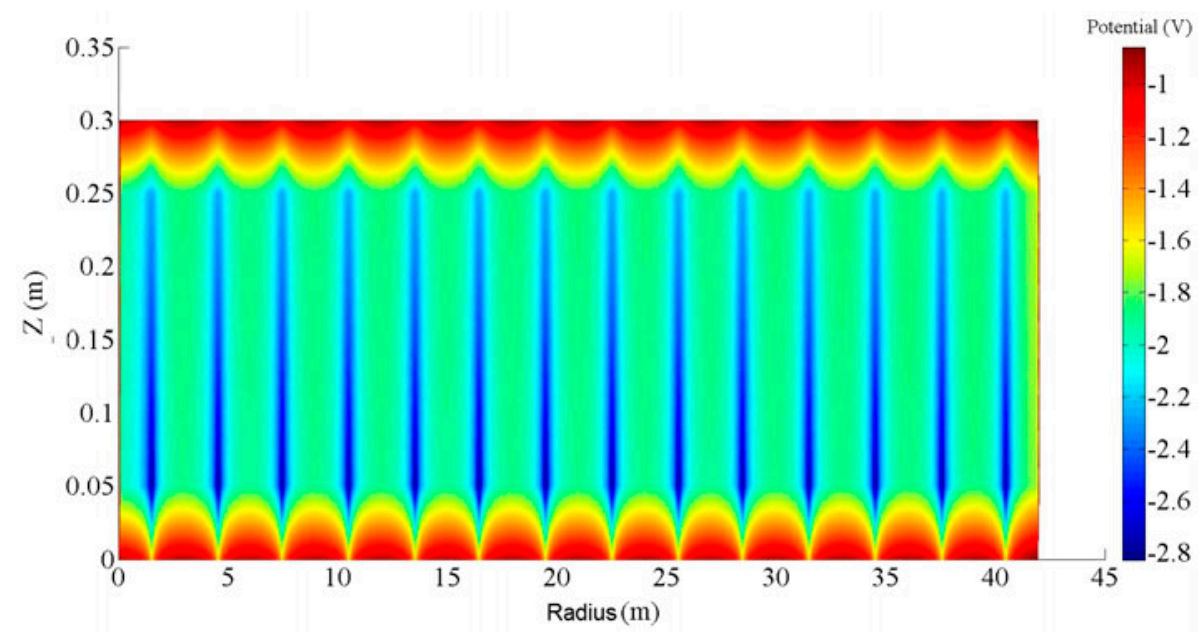

Figure 9. Potential in the soil layer.
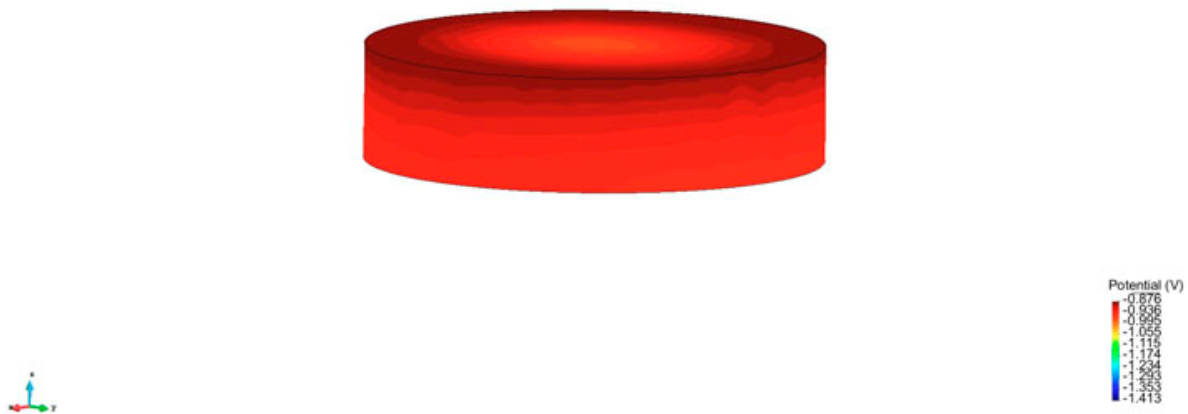

Figure 10. Potential distribution over the boundary of the soil layer - top view.

three anodes, close to the liner, within the electrolyte are considered. These anodes are mathematically represented as linear sources, therefore, a typical three-dimensional problem as shown in Figure 1 is studied. The height of the conductive concrete, the resistivity values and the polarisation curve are the same as in the previous example. Here, the radius of the tank bottom is equal to $1.0 \mathrm{~m}$ and each linear source has a length of $0.5 \mathrm{~m}$. The distance between the linear anodes is $0.25 \mathrm{~m}$.

The potential distribution along the boundary of the soil layer with resistivity $\rho_{2}$ is indicated in Figures 10 and 11. Table 3 shows the minimum and maximum potential values, computed and transposed to the tank bottom, using the modified polarisation curve determined by relation (13). The cylinder base (see Figure $1)$ is centred at $(0,0,0)$.

For such a real size example, in-situ electrochemical measurements have been performed to obtain the instant-off potential. These instant-off potentials, mea- 




$\sum_{0.1}^{2}$



Figure 11. Potential distribution over the boundary of the soil layer - bottom view.

Table 3. Maximum and minimum potential values.

\begin{tabular}{lcc}
\hline Location & Maximum potential $(\mathrm{V})$ & Minimum potential $(\mathrm{V})$ \\
\hline Interface & -0.876 & -0.978 \\
Metal & -0.873 & -0.972 \\
$(x, y)$ & $(-0.941,-0.080)$ & $(-0.007,-0.025)$ \\
\hline
\end{tabular}

sured immediately after switch-off of the $\mathrm{CP}$ current, are used as representative of the effective on-potential (with IR-drop compensation). The maximum and minimum potential values measured on site have been $-0.870 \mathrm{~V}$ and $-0.980 \mathrm{~V}$, respectively, indicating close enough agreement with the numerical simulation.

\section{Concluding remarks}

The main goal of this paper is to evaluate the design of CP systems of tank bottoms using an efficient boundary element procedure. In the scenario presented, the BEM implementation together with a subregion technique is commonly used to model the inhomogeneous domain. However, the numerical solution of this matrix system is usually the most time consuming step of the solution process, especially when the slender intermediate layer is to be discretised, requiring a considerable number of elements. Herein, a new methodology is proposed, a theoretically created polarisation curve at the common interface of adjacent zones is adopted, requiring no need for the implementation of the subregion technique.

Examples 1 and 2 show satisfactory equivalence between the proposed methodology and the alternative subregion technique, where the process time for the numerical calculation using the tested methodology was significantly less. In addition, simulations of cathodic protection systems show satisfactory estimates for the potential distribution over the metal to be protected involving practical problems such as bottoms of storage tanks, also including installation of secondary spill containments. 
In addition, most applications involving $\mathrm{CP}$ systems include optimisation and inverse problems. In such cases, the computer time difference, between the proposed methodology and the subregion technique, should be a lot more significant due to the necessity of repeatedly solving the numerical problem. These analyses should be the subject of future work.

\section{Acknowledgements}

The CAPES-Brazil, CNPq-Brazil and PEC/COPPE/UFRJ.

\section{Disclosure statement}

No potential conflict of interest was reported by the authors.

\section{ORCID}

W. J. Santos (D) http://orcid.org/0000-0001-5602-646X

J. A. F. Santiago (i) http://orcid.org/0000-0003-3089-954X

J. C. F. Telles (D) http://orcid.org/0000-0002-9940-4798

\section{References}

Abromowitz, M., \& Stegun, I. A. (1965). Handbook of mathematical functions. New York, NY: Dover.

Azevedo, J. P. S., \& Wrobel, L. C. (1988). Nonlinear heat conduction in composite bodies: A boundary element formulation. International Journal for Numerical Methods in Engineering, 26, 19-38. doi:10.1002/nme.1620260103

BEASY (2000). BEASY user guide. Southampton: Computational Mechanics BEASY Ltd.

Brasil, S. L. D. C., Telles, J. C. F., \& Miranda, L. R. M. (1991). On the effect of some critical parameters in cathodic protection systems: A numerical/experimental study. In R. S. Munn (Ed.), Computer Modeling in Corrosion, ASTM STP 1154 (pp. 277-291). Philadelphia, PA: American Society for Testing and Materials. doi:10.1520/STP24703S

Brebbia, C. A., \& Dominguez, J. (1989). Boundary elements: An introductory course. Southampton: Computational Mechanics Publications.

Brebbia, C. A., Telles, J. C. F., \& Wrobel, L. C. (1984). Boundary element techniques: Theory and applications in engineering. Berlin: Springer-Verlag.

Brebbia, C. A., \& Walker, S. (1980). Boundary element techniques in engineering. London: Butter-worths.

Fontana, M. G., \& Greene, N. D. (1967). Corrosion engineering. New York, NY: McGraw-Hill. ISO/15589-1 (2015). Petroleum, petrochemical and natural gas industries - Cathodic protection of pipeline systems. Part 1, On-land pipelines.

Kim, Y. S., Kim, J., Choi, D., Lim, J. Y., \& Kim, J. G. (2017). Optimizing the sacrificial anode cathodic protection of the rail canal structure in seawater using the boundary element method. Engineering Analysis with Boundary Elements, 77, 36-48. doi:10.1016/j.enganabound.2017.01.003

Kita, E., \& Kamiya, N. (1994). Subregion boundary element method. JSME International Journal Series A, Mechanics and Material Engineering, 37, 366-372. doi:10.1299/kikaia.59.415 
Koszewski, L. (1999). Retrofitting asphalt storage tanks with an improved cathodic protection system. Materials Performance, 38(6), 20-24.

Lu, X., \& Wu, W. (2005). A new subregion boundary element technique based on the domain decomposition method. Engineering Analysis with Boundary Elements, 29, 944952. doi:10.1016/j.enganabound.2005.08.001

Montoya, R., Aperador, W., \& Bastidas, D. M. (2009). Influence of conductivity on cathodic protection of reinforced alkali-activated slag mortar using the finite element method. Corrosion Science, 51, 2857-2862. doi:10.1016/j.corsci.2009.08.020

Montoya, R., Gakvan, J. C., \& Genesca, J. C. (2011). Using the right side of Poissons equation to save on numerical calculations in FEM simulation of electrochemical systems. Corrosion Science, 53, 1806-1812. doi:10.1016/j.corsci.2011.01.059

NACE/SP0169 (2007). Standard practice control of external corrosion on underground or submerged metallic piping systems.

Parsa, M. H., Allahkaram, S. R., \& Ghobadi, A. H. (2010). Simulation of cathodic protection potential distributions on oil well casings. Journal of Petroleum Science and Engineering, 72, 215-219. doi:10.1016/j.petrol.2010.03.020

Peabody, A. W. (2001). Peabody's control of pipeline corrosion (2nd ed.). Houston, TX: NACE International.

Riemer, D. P., \& Orazem, M. E. (2010). A mathematical model for the cathodic protection of tank bottoms. Corrosion Science, 47, 849-868. doi:10.1016/j.corsci.2004.07.018

Roberge, P. R. (1999). Handbook of corrosion engineering. New York, NY: McGraw-Hill.

Santiago, J. A. F., \& Telles, J. C. F. (1997). On boundary elements for simulation of cathodic protection systems with dynamic polarization curves. International Journal for Numerical Methods in Engineering, 40, 2611-2622. doi:10.1002/(SICI)1097-0207

Santos, W. J., Santiago, J. A. F., \& Telles, J. C. F. (2012). An application of genetic algorithms and the method of fundamental solutions to simulate cathodic protection systems. Computer Modeling in Engineering \& Sciences, 87, 23-40. doi:10.3970/cmes.2012.087.023

Santos, W. J., Santiago, J. A. F., \& Telles, J. C. F. (2014). Optimal positioning of anodes and virtual sources in the design of cathodic protection systems using the method of fundamental solutions. Engineering Analysis with Boundary Elements, 46, 67-74. doi:10.1016/j.enganabound.2014.05.009

Santos, W. J., Santiago, J. A. F., \& Telles, J. C. F. (2016). Using the Gaussian function to simulate constant potential anodes in multiobjective optimization of cathodic protection systems. Engineering Analysis with Boundary Elements, 73, 35-41. doi:10.1016/j.enganabound.2016.08.014

Telles, J. C. F., Mansur, W. J., Wrobel, L. C., \& Marinho, M. G. (1990). Numerical simulation of a cathodically protected semisubmersible platform using PROCAT system. Corrosion, 46, 513-518. doi:10.5006/1.3585141

US-EPA (1988). Title40 code of federal regulations, parts 280 and 281. September. 